\title{
Optimal Limited Stop-Loss Reinsurance under VaR, TVaR, and CTE Risk Measures
}

\author{
Xianhua Zhou, ${ }^{1}$ Huadong Zhang, ${ }^{2}$ and Qingquan Fan $^{3}$ \\ ${ }^{1}$ China Institute for Actuarial Science, Central University of Finance and Economics, Beijing 100081, China \\ ${ }^{2}$ Research Institute of Applied Mathematics, Anhua Agricultural Insurance Co., Ltd., Beijing 100037, China \\ ${ }^{3}$ School of Economics and Management, Tsinghua University, Beijing 100084, China
}

Correspondence should be addressed to Huadong Zhang; zhanghuadona@126.com

Received 27 April 2015; Revised 14 July 2015; Accepted 15 July 2015

Academic Editor: Xinguang Zhang

Copyright (C) 2015 Xianhua Zhou et al. This is an open access article distributed under the Creative Commons Attribution License, which permits unrestricted use, distribution, and reproduction in any medium, provided the original work is properly cited.

\begin{abstract}
This paper aims to provide a practical optimal reinsurance scheme under particular conditions, with the goal of minimizing total insurer risk. Excess of loss reinsurance is an essential part of the reinsurance market, but the concept of stop-loss reinsurance tends to be unpopular. We study the purchase arrangement of optimal reinsurance, under which the liability of reinsurers is limited by the excess of loss ratio, in order to generate a reinsurance scheme that is closer to reality. We explore the optimization of limited stoploss reinsurance under three risk measures: value at risk (VaR), tail value at risk (TVaR), and conditional tail expectation (CTE). We analyze the topic from the following aspects: (1) finding the optimal franchise point with limited stop-loss coverage, (2) finding the optimal limited stop-loss coverage within a certain franchise point, and (3) finding the optimal franchise point with limited stop-loss coverage. We provide several numerical examples. Our results show the existence of optimal values and locations under the various constraint conditions.
\end{abstract}

\section{Introduction}

Reinsurance, an agreement between insurers and reinsurers that allows insurers to transfer and diversify away a certain amount of risk, is the primary risk management tool used by insurance companies. The amount that an insurer pays to transfer risk to the reinsurer is known as the reinsurance premium. The losses caused by accidents that meet the requirements in the reinsurance contract (and that are borne by the reinsurer) are known as reinsurance recoverable. The insurer aims to reduce its compensation expenses to the greatest extent possible.

However, the situation is ever-changing, and optimal reinsurance has become a popular topic for both researchers and practitioners. This has resulted in a plethora of important insights. The earliest study on optimal reinsurance focused on safety loading, Borch [1]. A later article by Gerber [2] showed that excess of loss reinsurance is optimal at the expected value of the reinsurance principle. Gajek and Zagrodny [3] found that the optimal reinsurance form is the minimum variance under the standard deviation reinsurance principle, and Kaluszka [4] also showed that change loss reinsurance is optimal through mean-variance analysis of optimal reinsurance. Other researchers, such as $\mathrm{Bu}$ [5], have suggested that insurers wishing to determine the optimal trade-off between retained risk and expected profits should purchase middle layer reinsurance, because the purchase of reinsurance for high loss layers is not usually economically feasible. Cai and Tan [6] determined the optimal retention rate by minimizing VaR and CTE for insurers, while Cai et al. [7] found that the optimal reinsurance form is based on the principle of minimum VaR or CTE and that results differ under the two methods. Under CTE, excess of loss reinsurance is always the best product; under VaR, the answer is more complicated. Cai et al. [7] discussed this condition further for stop-loss reinsurance, but several issues remain, such as moral risk, overly high expenses, and a lack of stop-loss reinsurance products due to the extremely high risk for some types of insurance (e.g., crop insurance). The premium rates are relatively high because of the large reinsurance coverage, which in turn affects the insurance company's profits. Weng [8] attempts to fit an optimal reinsurance model in which 
reinsurance budget constraints are given, using the principles of minimum CTE and standard deviation reinsurance. He notes that, when the reinsurance premium is subject to a small budget, the best form is likely to be limited stoploss reinsurance, not standard stop-loss reinsurance. This is because, if the purchase reinsurance is insufficient, the primary insurer may become insolvent or even go bankrupt in the aftermath of several back-to-back catastrophes, Fu and Khury [9]. Chi and Tan [10] study the class of increasing convex ceded loss functions based on VaR and CVaR without specific forms of reinsurance. Their conclusions confirm the tenets of stop-loss reinsurance, except for Theorem 3.2 in [10]. To avoid the problem of moral hazards, Chi and Weng [11] conduct research under the Vajda condition, which stipulates that both the insurer's retained loss and the proportion paid by a reinsurer are increasing in indemnity. Other researchers, such as Gajek and Zagrodny [12] and Guerra and Centeno [13], prefer the theory of stop-loss reinsurance. More recent findings have been consistent with the notion that stop-loss reinsurance is the optimal product. Weng [8] and Porth et al. [14] add the reinsurance premium budget constraint to their model. The reinsurance premium equation must be solved when subject to the budget constraint, so the condition leads to a more complex problem. Therefore, we do not consider the reinsurance premium budget constraint here. Li et al. [15] study the optimal reinsurance and investment problem by capturing both the insurer's and the reinsurer's utility. Brandtner and Kürsten [16] investigate the problem of optimal reinsurance of risk management within the regulatory framework of Solvency II, under conditional VaR and spectral risk measures as its natural extension. Li et al. [15] modeled the risk process by Brownian motion with drift and studied the optimization problem of maximizing the exponential utility of terminal wealth under the controls of reinsurance and investment. Their results showed that optimal excess of loss reinsurance is generally a better product than optimal proportional reinsurance.

Thus, limited stop-loss reinsurance, which is the focus of this study, is the most practical real-world solution. In this study, we regard stop-loss reinsurance as a special case. We aim to solve the problem and provide optimal reinsurance advice to achieve optimal risk transfer under different risk measurement models and limiting conditions. The remainder of this paper is organized as follows. Section 2 presents the concepts and formulas related to reinsurance, as well as the formula of a reinsurance risk measurement model. Sections 3 and 4 explore optimal reinsurance arrangements and possible problems under VaR, TVaR, and CTE. Section 5 provides numerical examples and analyzes reinsurance premium constraints. Section 6 concludes.

\section{Basic Theory of Reinsurance and Risk Measures}

2.1. Limited Stop-Loss Reinsurance Theory. To illustrate the concept behind limited stop-loss reinsurance, consider an example involving coverage for the total amount of claim $X$ over one year. The reinsurer will pay the percentage of $X$ that exceeds a certain amount, for example, franchise point $d$.

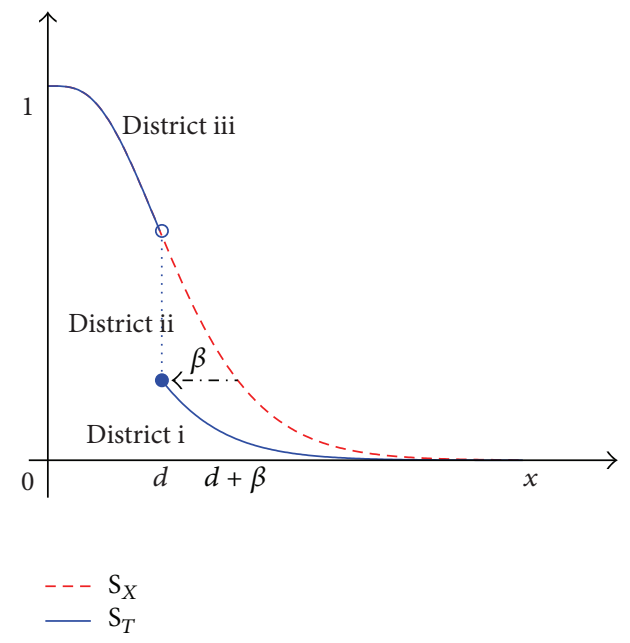

FIgURE 1: Relation between $S_{X}$ and $S_{I}$.

The reinsurer's liability is limited to that amount (e.g., limited stop-loss coverage $\beta)$. In general, $d \in(0, \infty)$ and $\beta \in(0, \infty)$. We let $X_{I}$ be the loss random variable of the cedent in the presence of limited stop-loss reinsurance, and we then have

$$
X_{I}= \begin{cases}X, & X<d, \\ d, & d \leq X \leq d+\beta, \\ X-\beta, & X>d+\beta .\end{cases}
$$

For stop-loss reinsurance, $\beta=\infty$. In other words, the reinsurer pays the excess of loss over $d$.

In this paper, subscripts $X, I$, and $T$ stand for the underlying loss, the retained loss, and the total loss of the cedent in the presence of limited stop-loss reinsurance. Survival function $S_{X}(x)=\operatorname{Pr}\{X>x\}=1-F_{X}(x)$, which is commonly used in actuarial science (for more details, see Cai and Tan [6]). Given that cumulative distribution function $F_{X}(x)$ is discontinuous, we use a strict inequality in survival function $S_{X}$ and in the risk measure. According to (1), we therefore have

$$
S_{I}= \begin{cases}S_{X}(x), & 0 \leq x<d \\ S_{X}(x+\beta), & x \geq d\end{cases}
$$

Figure 1 shows the relationship between $S_{X}$ and $S_{I}$, where $S_{I}$ is discontinuous. According to the jump of $S_{I}$, the entire plane is divided into three districts, as Figure 1 shows.

Let $\Pi(d, \beta)$ be the pure risk premium for the reinsurer as follows:

$$
\begin{aligned}
\Pi(d, \beta) & =\int_{d}^{d+\beta}(x-d) f_{X}(x) d x+\beta[1-F(d+\beta)] \\
& =\int_{d}^{d+\beta} S_{X}(x) d x
\end{aligned}
$$




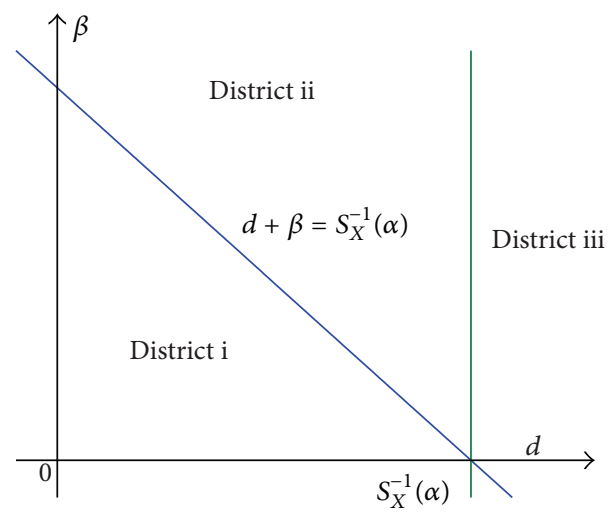

Figure 2: Plane $(d, \beta)$.

where $f_{X}(x)$ is the probability density function of $X$. Then, according to the expected value premium principle, the reinsurance premium $\delta(d, \beta)$ is

$$
\delta(d, \beta)=(1+\rho) \Pi(d, \beta),
$$

where $\rho>0$ is the safety loading. Hence, total risk $T$ is

$$
X_{T}=X_{I}+\delta(d, \beta) \text {. }
$$

2.2. Risk Measure after Reinsurance. In reinsurance studies, the risk measure is used to ensure optimal decision making (see, e.g., Cai et al. [7] and Bernard and Tian [17]). In risk management, several models, such as VaR, CTE, TVaR, CVaR, and ES, are commonly used. Within a certain time period and at a confidence level of $1-\alpha, 0<\alpha<1$, the highest risk value of $X$ does not exceed VaR; CTE is the expected value of events that occur outside the probability alpha. According to the consistency axiom of risk measure defined by Artzner et al. [18], several of these risk measures do not satisfy the consistency axiom. For example, VaR does not meet the additivity condition.

According to Hang [19] and the combined characteristic of $X_{I}$, we have

$$
\begin{aligned}
& \operatorname{VaR}_{I}(d, \alpha, \beta)=\inf \left\{x \mid \operatorname{Pr}\left(X_{I}>x\right) \leq \alpha\right\} \\
& =\inf \left\{x \mid S_{I}(x) \leq \alpha\right\} \\
& = \begin{cases}S_{X}^{-1}(\alpha)-\beta, & 0<d+\beta<S_{X}^{-1}(\alpha), \text { i, } \\
d, & d \leq S_{X}^{-1}(\alpha) \leq d+\beta, \text { ii, } \\
S_{X}^{-1}(\alpha), & S_{X}^{-1}(\alpha)<d, \text { iii. }\end{cases}
\end{aligned}
$$

For conciseness, we denote $\mathrm{VaR}_{T}^{\mathrm{i}}$ as $\mathrm{VaR}_{T}$ in district $\mathrm{i}$ throughout the remainder of paper; the variables with no subscript stand for those in the entire district. The rest of the variables are similar. $S_{I}$ is divided into three districts. Similarly, we divide plane $(d, \beta)$ into three districts: $i$, ii, and iii (Figure 2). The boundaries of $\mathrm{i}$ and ii and of ii and iii are classified in ii.

From (5) and (6), we therefore have

$$
\operatorname{VaR}_{T}(d, \alpha, \beta)=\operatorname{VaR}_{I}(d, \alpha, \beta)+\delta(d, \beta),
$$

where $\operatorname{VaR}_{I}$ is continuous on the plane and $\delta(d, \beta)$ is continuous. Hence, $\mathrm{VaR}_{T}$ is also continuous on the plane.

From (5) and (7), we can easily prove $S_{T}\left(\operatorname{VaR}_{T}(d, \alpha, \beta)\right)=$ $S_{I}\left(\operatorname{VaR}_{I}(d, \alpha, \beta)\right)$. In district $\mathrm{i}$, we have $0<d+\beta<S_{X}^{-1}(\alpha)$, and $\operatorname{VaR}_{I}(d, \alpha, \beta)=S_{X}^{-1}(\alpha)-\beta>d$. With (2), we have

$$
\begin{aligned}
& S_{I}\left(\operatorname{VaR}_{I}(d, \alpha, \beta)\right)=S_{I}\left(S_{X}^{-1}(\alpha)-\beta\right)=\alpha, \\
& E\left[\left(X_{I}-\operatorname{VaR}_{I}(d, \alpha, \beta)\right)^{+}\right] \\
& \quad=\int_{\operatorname{VaR}_{I}(d, \alpha, \beta)}^{\infty}\left(x-\beta-\operatorname{VaR}_{I}(d, \alpha, \beta)\right) f_{X}(x) d x \\
& \quad=\int_{S_{X}^{-1}(\alpha)}^{\infty} S_{X}(x) d x .
\end{aligned}
$$

In district ii, we have $d \leq S_{X}^{-1}(\alpha) \leq d+\beta$ and $\operatorname{VaR}_{I}(d, \alpha, \beta)=$ $d$. With (2), we therefore have

$$
\begin{aligned}
& S_{I}\left(\operatorname{VaR}_{I}(d, \alpha, \beta)\right)=S_{I}(d)=S_{X}(d+\beta), \\
& E\left[\left(X_{I}-\operatorname{VaR}_{I}(d, \alpha, \beta)\right)^{+}\right] \\
& \quad=\int_{d+\beta}^{\infty}(x-\beta-d) f_{X}(x) d x=\int_{d+\beta}^{\infty} S_{X}(x) d x .
\end{aligned}
$$

In district iii, we have $S_{X}^{-1}(\alpha)<d$ and $\operatorname{VaR}_{I}(d, \alpha, \beta)=$ $S_{X}^{-1}(\alpha)<d$. With (2), we have

$$
\begin{aligned}
& S_{I}\left(\operatorname{VaR}_{I}(d, \alpha, \beta)\right)=S_{I}\left(S_{X}^{-1}(\alpha)\right)=\alpha, \\
& E\left[\left(X_{I}-\operatorname{VaR}_{I}(d, \alpha, \beta)\right)^{+}\right] \\
& =\int_{S_{X}^{-1}(\alpha)}^{d}\left(x-S_{X}^{-1}(\alpha)\right) f_{X}(x) d x \\
& \quad+\left[d-S_{X}^{-1}(\alpha)\right]\left[F_{X}(d+\beta)-F_{X}(d)\right] \\
& \quad+\int_{d+\beta}^{\infty}\left(x-\beta-S_{X}^{-1}(\alpha)\right) f_{X}(x) d x \\
& =\int_{S_{X}^{-1}(\alpha)}^{d} S_{X}(x) d x+\int_{d+\beta}^{\infty} S_{X}(x) d x .
\end{aligned}
$$

We obtain the expression of ES as follows:

$$
\begin{aligned}
& \mathrm{ES}_{I}(d, \alpha, \beta)=E\left[\left(X_{I}-\operatorname{VaR}_{I}(d, \alpha, \beta)\right)^{+}\right] \\
& \quad= \begin{cases}\int_{S_{X}^{-1}(\alpha)}^{\infty} S_{X}(x) d x, & \mathrm{i}, \\
\int_{S_{X}^{-1}(\alpha)}^{\infty} S_{X}(x) d x-\int_{S_{X}^{-1}(\alpha)}^{d+\beta} S_{X}(x) d x, & \text { ii, } \\
\int_{S_{X}^{-1}(\alpha)}^{\infty} S_{X}(x) d x-\int_{d}^{d+\beta} S_{X}(x) d x, & \text { iii. }\end{cases}
\end{aligned}
$$


And, with (5) and (6), we obtain

$$
\begin{aligned}
\mathrm{ES}_{T}(d, \alpha, \beta) & =E\left[\left(X_{T}-\operatorname{VaR}_{T}(d, \alpha, \beta)\right)^{+}\right] \\
& =E\left[\left(X_{I}-\operatorname{VaR}_{I}(d, \alpha, \beta)\right)^{+}\right] \\
& =\mathrm{ES}_{I}(d, \alpha, \beta) .
\end{aligned}
$$

According to the transformation among risk measure models (Hang [19] and Charpentier [20]), we have

$$
\begin{aligned}
& \operatorname{TVaR}_{I}(d, \alpha, \beta)=\inf _{a>0}\left\{a+\frac{1}{\alpha} E\left[\left(X_{I}-a\right)^{+}\right]\right\} \\
& =\operatorname{VaR}_{I}(d, \alpha, \beta)+\frac{1}{\alpha} \mathrm{ES}_{I}(d, \alpha, \beta), \\
& \operatorname{CVaR}_{I}(d, \alpha, \beta) \\
& =E\left[X_{I}-\operatorname{VaR}_{I}(d, \alpha, \beta) \mid X_{I}>\operatorname{VaR}_{I}(d, \alpha, \beta)\right] \\
& =\frac{1}{S_{I}\left(\operatorname{VaR}_{I}(d, \alpha, \beta)\right)} \mathrm{ES}_{I}(d, \alpha, \beta), \\
& \mathrm{CTE}_{I}(d, \alpha, \beta)=E\left[X X_{I} \mid X_{I}>\operatorname{VaR}_{I}(d, \alpha, \beta)\right] \\
& =\operatorname{VaR}_{I}(d, \alpha, \beta) \\
& \quad+\frac{1}{S_{I}\left(\operatorname{VaR}_{I}(d, \alpha, \beta)\right)} \mathrm{ES}_{I}(d, \alpha, \beta) .
\end{aligned}
$$

Obviously, TVaR, CTE, and CVaR can all be obtained by combining VaR, ES, $S$, and $\alpha$. Equations (7) and (12) denote the transforming relationships between $X_{I}$ and $X_{T}$ of VaR and ES, respectively. Hence, no expressions of TVaR, CTE, or CVaR exist in $X_{T}$. For information risk measures, see Charpentier [20].

We define risk measures in this paper as per Hang [19], whose definitions are slightly different from those proposed by Wirch and Hardy [21]. According to $S_{T}=S_{I}$ and (12), ES and CVaR models do not include the information of reinsurance premium $\delta(d, \beta)$. Thus, they are not suitable for making reinsurance decisions, and no further analysis of them is needed in this context.

Under risk measure $\Lambda$ and condition $\Theta$, the aim is to solve the optimization problem

$$
\min _{\theta \in \Theta} \Lambda_{T}(\theta)
$$

Note that, in this study, $\theta=(d, \alpha, \beta)$ and $\Lambda \in\{\mathrm{VaR}$, TVaR, $\mathrm{CTE}\}$, but $\Theta$ has different expressions.

\section{Optimal Reinsurance under the VaR Risk Measure}

We let $\Lambda=\mathrm{VaR}$, the most commonly used and concise risk measure in risk management. From (3), (4), (6), and (7), we therefore have

$$
\frac{\partial \operatorname{VaR}_{T}(d, \alpha, \beta)}{\partial d}
$$

$$
= \begin{cases}(1+\rho)\left(S_{X}(d+\beta)-S_{X}(d)\right)<0, & \text { i, } \\ 1+(1+\rho)\left(S_{X}(d+\beta)-S_{X}(d)\right), & \text { ii, } \\ (1+\rho)\left(S_{X}(d+\beta)-S_{X}(d)\right)<0, & \text { iii, }\end{cases}
$$

$$
\frac{\partial^{2} \operatorname{VaR}_{T}(d, \alpha, \beta)}{\partial d^{2}}=(1+\rho)\left(f_{X}(d)-f_{X}(d+\beta)\right)
$$

$$
(d, \beta) \in \mathrm{i}, \mathrm{ii}, \mathrm{iii},
$$

$$
\begin{gathered}
\frac{\partial \operatorname{VaR}_{T}(d, \alpha, \beta)}{\partial \beta}=\left\{\begin{array}{l}
-1+(1+\rho) S_{X}(d+\beta), \quad \text { i, } \\
(1+\rho) S_{X}(d+\beta)>0, \\
(1+\rho) S_{X}(d+\beta)>0,
\end{array} \quad\right. \text { ii, } \\
\frac{\partial^{2} \operatorname{VaR}_{T}(d, \alpha, \beta)}{\partial \beta^{2}}=-(1+\rho) f_{X}(d+\beta)<0, \\
\quad(d, \beta) \in \text { i, ii, iii, } \\
\frac{\partial^{2} \operatorname{VaR}_{T}(d, \alpha, \beta)}{\partial d \partial \beta}=-(1+\rho) f_{X}(d+\beta)<0, \\
\quad(d, \beta) \in \text { i, ii, iii. }
\end{gathered}
$$

In any district, $\mathrm{VaR}_{T}$ is monotonous for either $d$ or $\beta$. In other words, it cannot form a stagnation point. Therefore, the optimal solution can only exist on the border.

3.1. Optimal $d$ for Given $\beta$. At any given point in time on the reinsurance market, some reinsurance policies are not active. For example, the coverage of reinsurance in the market is generally given as $\beta=0.2,0.3$. For management purposes, company executives typically provide a definite coverage amount in advance, with the goal of finding the optimal franchise point for given reinsurance coverage $\beta$ that will minimize total insurer risk. We let $\Theta=\{d \mid d>0\}$. Therefore, the optimization problem must be addressed as follows:

$$
\min _{d>0} \operatorname{VaR}_{T}(d, \alpha, \beta)
$$

As we noted previously, $\mathrm{VaR}_{T}$ is continuous on the entire plane. From (15), we know that $\mathrm{VaR}_{T}$ is decreasing in $\mathrm{i}$ and iii, and

$$
\operatorname{VaR}_{T}(\infty, \alpha, \beta)=\lim _{d \rightarrow \infty} \operatorname{VaR}_{T}(d, \alpha, \beta)=S_{X}^{-1}(\alpha),
$$


where $\operatorname{VaR}_{T}(\infty, \alpha, \beta)$ represents the case where $d$ approaches infinity and $\operatorname{VaR}_{T}^{\mathrm{ii}}(0, \alpha, \beta)$ represents 0 . For the sake of space, we do not provide any detailed demonstrations here, however.

Next, we establish that

$$
\begin{aligned}
& h(\beta)=(1+\rho) \int_{S_{X}^{-1}(\alpha)-\beta}^{S_{X}^{-1}(\alpha)} S_{X}(x) d x-\beta, \\
& \qquad 0 \leq \beta \leq S_{X}^{-1}(\alpha), \\
& h^{\prime}(\beta)=(1+\rho) S_{X}\left(S_{X}^{-1}(\alpha)-\beta\right)-1, \\
& h^{\prime \prime}(\beta)=(1+\rho) f_{X}\left(S_{X}^{-1}(\alpha)-\beta\right)>0 .
\end{aligned}
$$

Note that $h$ is a strictly convex function in $\beta$. By letting $h^{\prime}(\beta)=$ 0 , we can solve

$$
\tilde{\beta}=S_{X}^{-1}(\alpha)-S_{X}^{-1}\left(\frac{1}{1+\rho}\right) .
$$

We let $\widetilde{\beta}>0$; that is,

$$
\alpha *(1+\rho)<1,
$$

while, at the same time, $h(0)=0$. By letting $h\left(\beta^{*}\right)=0$, we can obtain the numerical solution of $\beta^{*}$. Thus, $\beta^{*}>\widetilde{\beta}$ under the condition of (20), and we have $\forall \beta \in\left[0, \beta^{*}\right], h(\beta) \leq 0$.

Note that (20) is very important, because, in general, safety loading $\rho$ cannot be too large, or the risk distribution will be unsuitable. If $\rho=1$, then $\alpha<0.5$; if $\rho=0.5$, then $\alpha<2 / 3$. When the general consideration of $\alpha$ is relatively small and if the insurance company's risk tolerance level $\alpha=$ 0.5 , then it will be unsuitable for the excess of loss ratio reinsurance.

Let $d^{*}$ be the solution of

$$
S_{X}(d)-S_{X}(d+\beta)=\frac{1}{1+\rho} .
$$

In this manner, if $\left(d^{*}, \beta\right) \in \mathrm{ii}$, then

$$
\left.\frac{\partial \operatorname{VaR}_{T}(d, \alpha, \beta)}{\partial d}\right|_{\left(d^{*}, \beta\right) \in \mathrm{ii}}=0 .
$$

The existence and uniqueness of $d^{*}$ depends on the distribution of $f_{X}$ and $\rho$. We define $\stackrel{\dot{d}}{=}=\inf \left\{d: \forall \beta>0, f_{X}(d)>\right.$ $\left.f_{X}(d+\beta)\right\}$. In fact, $d$ is the rightmost peak of risk distribution $f_{X}$.

Lemma 1. If (20) is satisfied, then $\forall \beta \in\left(0, \beta^{*}\right), V a R_{T}$ can achieve the optimum, and franchise $d$ should be in district ii. Furthermore, (1) if $\beta \leq S_{X}^{-1}(\alpha)-\dot{d}$ and $d^{*}>S_{X}^{-1}(\alpha)-\beta$, then one can find the optimal $V a R_{T}$ in $d=d^{*}$ and (2) if $\beta \leq S_{X}^{-1}(\alpha)-\stackrel{\circ}{d}$ and $d^{*} \leq S_{X}^{-1}(\alpha)-\beta$, one can find the optimal $V R_{T}$ in $d=$ $S_{X}^{-1}(\alpha)-\beta$.

Proof. As mentioned in the previous sections, districts $i$ and iii are open sets. However, $\mathrm{VaR}_{T}$ is monotonically decreasing

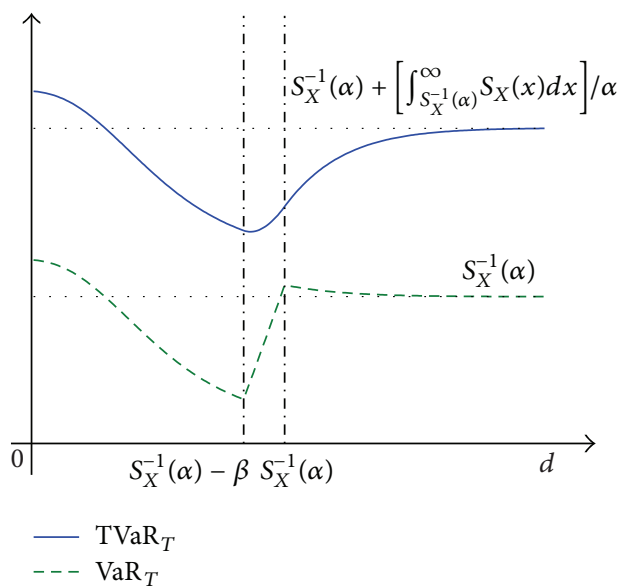

Figure 3: When $\beta$ is given, $\operatorname{VaR}_{T}$ and $\operatorname{TVaR}_{T}$ satisfy (20).

in $d$. Therefore, if an optimal $\mathrm{VaR}_{T}$ exists, it must be the optimal $\mathrm{VaR}_{T}^{\mathrm{ii}}$. In boundary district ii, $d=S_{X}^{-1}(\alpha)-\beta$, so, from (6), (7), and (20), we have

$$
\operatorname{VaR}_{T}\left(S_{X}^{-1}(\alpha)-\beta, \alpha, \beta\right)-\operatorname{VaR}_{T}(\infty, \alpha, \beta)=h(\beta) .
$$

Moreover, as we can deduce from $(20), h(\beta) \leq 0$. Thus, we know that $\operatorname{VaR}_{T}\left(S_{X}^{-1}(\alpha)-\beta, \alpha, \beta\right)$ is not larger than $\operatorname{VaR}_{T}(\infty, \alpha, \beta)$. In a given situation $\beta$, district ii about $d$ is a closed set, so $\mathrm{VaR}_{T}$ in district ii will attain an optimal solution.

When $\beta \leq S_{X}^{-1}(\alpha)-\stackrel{\circ}{d}, d \geq \stackrel{\circ}{d}$. With (15), we know that $\mathrm{VaR}_{T}^{\mathrm{ii}}$ about $d$ is convex, which means that the optima of $\mathrm{VaR}_{T}^{\mathrm{ii}}$ are found at extreme points where the first derivative of $\mathrm{VaR}_{T}^{\mathrm{ii}}$ is 0 . If $d^{*} \geq S_{X}^{-1}(\alpha)$, then $\operatorname{VaR}_{T}^{\text {ii }}$ is monotonically decreasing in $\left[S_{X}^{-1}(\alpha)-\beta, S_{X}^{-1}(\alpha)\right]$. However, $\operatorname{VaR}_{T}$ on $d=S_{X}^{-1}(\alpha)$ is continuous, and it will begin to decrease from this point to $\operatorname{VaR}_{T}(\infty, \alpha, \beta)$. This condition is contrary to that found under $h(\beta) \leq 0$. Hence, under the condition of $(20), d^{*}<S_{X}^{-1}(\alpha)$.

If $d^{*}>S_{X}^{-1}(\alpha)-\beta$ and $d^{*} \in\left(S_{X}^{-1}(\alpha)-\beta, S_{X}^{-1}(\alpha)\right)$, $\mathrm{VaR}_{T}$ on $d=d^{*}$ will exist as an optimal solution. When $d^{*} \leq S_{X}^{-1}(\alpha)-\beta, \operatorname{VaR}_{T}^{\mathrm{ii}}$ on $d$ is increasing; therefore, when $d=S_{X}^{-1}(\alpha)-\beta$, we can find the optimal solution, which is also the optimal $\mathrm{VaR}_{T}$. Figure 3 gives a graphical representation of $\min _{d>0} \operatorname{VaR}_{T}(d, \alpha, \beta)$.

The proof indicates the existence of $d^{*}$ and implies that the relationship between $d^{*}$ and $\dot{d}$ only affects the position of the optimal solution, not its existence.

3.2. Optimal $\beta$ for Given $d$. Note that insurance companies may consider a reinsurance position where the franchise point is the profit equilibrium point, because they can obtain a certain profit before the threshold. Beyond this threshold, profits will be reduced or even reach a deficit. Thus, the best option is appropriate reinsurance coverage $\beta$ to achieve optimality. $d$ is constant, and $\beta$ is a variable. The optimization problem is $\Lambda=\operatorname{VaR}, \Theta=\{\beta \mid \beta>0\}$. 
Next, we let

$$
\begin{aligned}
& g(d)=d+(1+\rho) \int_{d}^{S_{X}^{-1}(\alpha)} S_{X}(x) d x-S_{X}^{-1}(\alpha), \\
& \quad 0 \leq d \leq S_{X}^{-1}(\alpha), \\
& g^{\prime}(d)=1-(1+\rho) S_{X}(d), \\
& g^{\prime \prime}(d)=(1+\rho) f_{X}(d)>0,
\end{aligned}
$$

where $g$ is a strictly convex function in $d$. The solution of $g^{\prime}(d)=0$ is $\tilde{d}$ :

$$
\begin{aligned}
& \widetilde{d}=S_{X}^{-1}\left(\frac{1}{1+\rho}\right), \\
& \widetilde{d}<S_{X}^{-1}(\alpha) \longleftrightarrow(20) .
\end{aligned}
$$

Furthermore, $g\left(S_{X}^{-1}(\alpha)\right)=0$. Hence, under (20), another solution is $\widetilde{d^{*}}<S_{X}^{-1}(1 /(1+\rho))$, which can be obtained by using a numerical method. For $\forall d \in\left[\widetilde{d^{*}}, S_{X}^{-1}(\alpha)\right]$, we have $g(d) \leq 0$.

Lemma 2. If (20) is satisfied, $\forall d \in\left[\widetilde{d^{*}}, S_{X}^{-1}(\alpha)\right]$. VaR $R_{T}$ can then obtain the optimal value when $\beta=S_{X}^{-1}(\alpha)-d$ :

$$
\begin{aligned}
& \operatorname{VaR}_{T}\left(d, \alpha, S_{X}^{-1}(\alpha)-d\right) \\
& \quad=d+(1+\rho) \int_{d}^{S_{X}^{-1}(\alpha)} S_{X}(x) d x .
\end{aligned}
$$

$\forall d \notin\left[\widetilde{d^{*}}, S_{X}^{-1}(\alpha)\right]$, and no optimal solutions of $\beta$ exist in $V a R_{T}$.

Proof. According to (15), when $d>S_{X}^{-1}(\alpha)$ (district iii, open set), then $\mathrm{VaR}_{T}$ will be increasing with regard to $\beta$; hence, no optimal value exists. However, when $\beta \geq S_{X}^{-1}(\alpha)-d$ (district ii, half closed), $\operatorname{VaR}_{T}$ will be increasing with regard to $\beta$, and the minimum will be reached at the lower boundary. $\operatorname{VaR}_{T}$ in district $i$ is convex with regard to $\beta$. Thus, if the value obtained on the boundary of districts $i$ and ii is not greater than the lower limit value, then we can obtain the optimal value, or the combination of properties of the convex function will indicate no optimal value.

$$
\begin{aligned}
& \forall d \in\left[\widetilde{d^{*}}, S_{X}^{-1}(\alpha)\right], \text { and let } \beta=S_{X}^{-1}(\alpha)-d: \\
& \operatorname{VaR}_{T}\left(d, \alpha, S_{X}^{-1}(\alpha)-d\right)-\operatorname{VaR}_{T}(d, \alpha, \infty)=g(d) \\
& \quad<0 .
\end{aligned}
$$

The boundary of districts $\mathrm{i}$ and ii $d+\beta=S_{X}^{-1}(\alpha)$. Given that the value of $\operatorname{VaR}_{T}$ is less than the limit value of $S_{X}^{-1}(\alpha)$, this is optimal. However, $\forall d<\widetilde{d^{*}}, g(d)>0$, so no optimal solution exists. Figure 4 gives a graphical representation of $\min _{\beta>0} \operatorname{VaR}_{T}(d, \alpha, \beta)$.

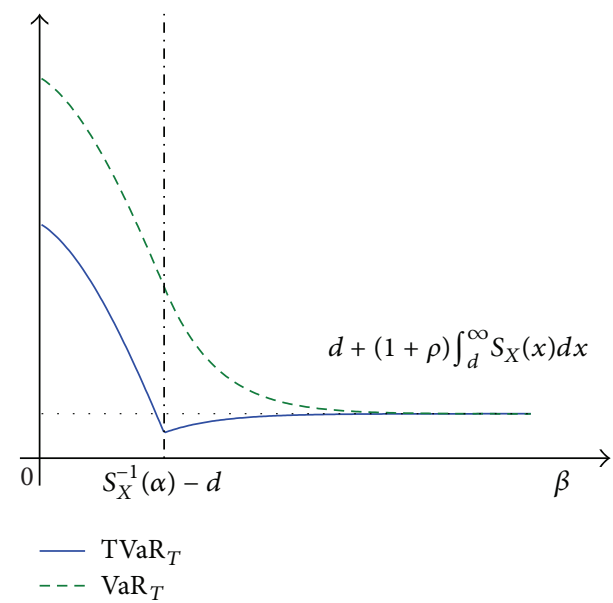

FIgURE 4: When $d$ is given, $\operatorname{VaR}_{T}$ and $\mathrm{TVaR}_{T}$ satisfy (20).

3.3. Comprehensive Effect. After analyzing the optimal decision under given franchise point $d$ or given reinsurance coverage $\beta$, we consider comprehensive effects with two variables. The optimization problem is $\Lambda=\operatorname{VaR}, \Theta=\{(d, \beta) \mid$ $d>0, \beta>0\}$. The following analysis is under a function of $d$, and we obtain similar results if we perform it under a function of $\beta$.

Lemma 3. Under the condition of (20), $\mathrm{VaR}_{T}$ can obtain the global optimal value on $\left(\widetilde{d}, S_{X}^{-1}(\alpha)-\widetilde{d}\right)$.

Proof. The optimal value of $\mathrm{VaR}_{T}$ only occurs on the border of districts $\mathrm{i}$ and ii. Lemma 2 ensures that $\operatorname{VaR}_{T}(d, \alpha, \beta) \leq$ $S_{X}^{-1}(\alpha)$ under the condition of $(20)$ is not empty. We let $\forall(d, \beta): d+\beta=S_{X}^{-1}(\alpha)$, and

$$
\begin{aligned}
\operatorname{VaR}_{T}(d, \alpha, \beta) & =d+(1+\rho) \int_{d}^{S_{X}^{-1}(\alpha)} S_{X}(x) d x \\
\frac{\partial \operatorname{VaR}_{T}(d, \alpha, \beta)}{\partial d} & =1-(1+\rho) S_{X}(d) \\
\frac{\partial^{2} \operatorname{VaR}_{T}(d, \alpha, \beta)}{\partial d^{2}} & =(1+\rho) f_{X}(d)>0 .
\end{aligned}
$$

$\mathrm{VaR}_{T}$ is convex in the border district, and we obtain the minimum value when the first derivative is 0 . We do not consider $d=0$ or $\infty$ :

$$
\frac{\partial \operatorname{VaR}_{T}(d, \alpha, \beta)}{\partial d}=0 \longleftrightarrow d=S_{X}^{-1}\left(\frac{1}{1+\rho}\right) .
$$

The value of $\widetilde{d}$ falls within the district, as required by Lemma 2. Thus, under the condition of (20), $\mathrm{VaR}_{T}$ attains the global optimal value on $\left(\tilde{d}, S_{X}^{-1}(\alpha)-\widetilde{d}\right)$.

Given a company's risk preference $\alpha$ and safety loading $\rho$, we can obtain the optimal solution on $\tilde{d}=S_{X}^{-1}(1 /(1+\rho))$, $\widetilde{\beta}=S_{X}^{-1}(\alpha)-S_{X}^{-1}(1 /(1+\rho))$. An undesirable phenomenon thus exists: risk preference $\alpha$ cannot affect the optimal franchise 
point. It can only affect the reinsurance coverage. Chi and Tan [10] set no constraint on $\beta=0$ or $\beta=\infty$. Theorem 3.2 in [10] provides the same conclusion as Lemma 3.

\section{Optimal Reinsurance under TVaR and CTE Risk Measures}

4.1. Under TVaR Risk Measures. In this subsection, $\Lambda=$ TVaR. Equation (13) provides the TVaR formula, and $\mathrm{VaR}_{T}$ and $\mathrm{ES}_{T}$ are both continuous on the entire plane. Thus, $\mathrm{TVaR}_{T}$ is also continuous. According to (13) and (15), we have

$$
\begin{aligned}
& \frac{\partial \operatorname{TVaR}_{T}(d, \alpha, \beta)}{\partial d} \\
& =\frac{\partial \operatorname{TVaR}_{T}(d, \alpha, \beta)}{\partial d}+\frac{1}{\alpha} \frac{\partial \mathrm{ES}_{T}(d, \alpha, \beta)}{\partial d} \\
& = \begin{cases}(1+\rho)\left(S_{X}(d+\beta)-S_{X}(d)\right)<0, & \mathrm{i}, \\
1-\left[\frac{1}{\alpha}-(1+\rho)\right] S_{X}(d+\beta)-(1+\rho) S_{X}(d), & \mathrm{ii}, \\
{\left[\frac{1}{\alpha}-(1+\rho)\right]\left(S_{X}(d)-S_{X}(d+\beta)\right),} & \mathrm{iii},\end{cases} \\
& \frac{\partial \operatorname{TVaR}_{T}(d, \alpha, \beta)}{\partial \beta}= \begin{cases}-1+(1+\rho) S_{X}(d+\beta), & \text { i, } \\
-\left[\frac{1}{\alpha}-(1+\rho)\right] S_{X}(d+\beta), & \text { ii, } \\
-\left[\frac{1}{\alpha}-(1+\rho)\right] S_{X}(d+\beta), & \text { iii. }\end{cases}
\end{aligned}
$$

For a given $\beta$, TVaR is monotonically decreasing in district $\mathrm{i}$, and, under the condition of (20), it is monotonically increasing in district iii. Hence, an optimal solution exists in district ii.

Lemma 4. Under the condition of (20) and (1) $\forall \beta>0(\Theta=$ $\{d \mid d>0\})$, the optimal solution of TVaR appears in district ii; (2) in the case of $d(\Theta=\{\beta \mid \beta>0\})$, no optimal value of TVaR exists; and (3) no global optimal value of TVaR $(\Theta=$ $\{(d, \beta) \mid d>0, \beta>0\})$ exists.

Proof. Figure 3 gives a graphical representation of $\min _{d>0} \operatorname{TVaR}_{T}(d, \alpha, \beta)$. At a given $d$,

$$
\frac{\partial^{2} \operatorname{TVaR}_{T}^{\mathrm{i}}(d, \alpha, \beta)}{\partial \beta^{2}}=-(1+\rho) f_{X}(d+\beta)<0
$$

Therefore, in district $\mathrm{i}$, the $\mathrm{TVaR}_{T}$ of $\beta$ is convex. However, under (20), $\mathrm{TVaR}_{T}$ in districts ii and iii of $\beta$ is decreasing, so no optimal solution exists (Figure 3 ). In this case, we cannot obtain an optimal solution by only changing $\beta$. And, moreover, no optimal solution exists when we change $d$ and $\beta$.

4.2. Under CTE Risk Measures. In this subsection, $\Lambda=\mathrm{CTE}$. According to (13) and so on, we have

$$
\begin{aligned}
\mathrm{CTE}_{T}(d, \alpha, \beta)= & \operatorname{VaR}_{T}(d, \alpha, \beta)+\mathrm{CVaR}_{T}(d, \alpha, \beta) \\
= & \left\{\begin{array}{l}
S_{X}^{-1}(\alpha)-\beta+(1+\rho) \int_{d}^{d+\beta} S_{X}(x) d x+\frac{1}{\alpha} \int_{S_{X}^{-1}(\alpha)}^{\infty} S_{X}(x) d x, \\
d+(1+\rho) \int_{d}^{d+\beta} S_{X}(x) d x+\frac{1}{S_{X}(d+\beta)} \int_{d+\beta}^{\infty} S_{X}(x) d x, \\
S_{X}^{-1}(\alpha)+\frac{1}{\alpha} \int_{S_{X}^{-1}(\alpha)}^{\infty} S_{X}(x) d x-\left[\frac{1}{\alpha}-(1+\rho)\right] \int_{d}^{d+\beta} S_{X}(x) d x,
\end{array}\right.
\end{aligned}
$$

Lemma 5. CTE $E_{T}$ is continuous between districts $i$ and ii and discontinuous between districts ii and iii. One therefore has:

$$
\operatorname{CTE}_{T}^{i i}\left(S_{X}^{-1}(\alpha), \alpha, \beta\right)>C T E_{T}^{i i i}\left(S_{X}^{-1}(\alpha), \alpha, \beta\right)
$$

Proof. $\mathrm{VaR}_{T}$ and $\mathrm{ES}_{T}$ are continuous on the plane, so we only need to prove the numerator of $\mathrm{CTE}_{T}$. If $\forall\left(d_{0}, \beta_{0}\right): d_{0}+\beta_{0}=$ $S_{X}^{-1}(\alpha)$, then $\left(d_{0}, \beta_{0}\right) \in \mathrm{ii}$; it is in the boundary of districts $\mathrm{i}$ and ii. We have $S_{X}\left(d_{0}+\beta_{0}\right)=\alpha$. Hence, $\mathrm{CTE}_{T}$ is continuous between districts $\mathrm{i}$ and ii.

If $\forall \beta_{0}>0, d_{0}=S_{X}^{-1}(\alpha)$, then $\left(d_{0}, \beta_{0}\right) \in \mathrm{ii}$; it is on the boundary of districts ii and iii. We have $1 / S_{X}\left(d_{0}+\beta_{0}\right)>$ $1 / \alpha$. Thus, $\mathrm{CTE}_{T}$ is discontinuous between districts ii and iii. Furthermore, $\mathrm{CTE}_{T}^{\mathrm{ii}}\left(S_{X}^{-1}(\alpha), \alpha, \beta\right)>\mathrm{CTE}_{T}^{\mathrm{iii}}\left(S_{X}^{-1}(\alpha), \alpha, \beta\right)$.
From (15) and (33), we have

$$
\begin{aligned}
& \frac{\partial \mathrm{CTE}_{T}}{\partial d} \\
& = \begin{cases}-(1+\rho)\left(S_{X}(d)-S_{X}(d+\beta)\right)<0, & \mathrm{i}, \\
-(1+\rho)\left(S_{X}(d)-S_{X}(d+\beta)\right)+\frac{f_{X}(d+\beta)}{S_{X}(d+\beta)} \mathrm{CVaR}_{T}^{\mathrm{ii}}, & \mathrm{ii}, \\
{\left[\frac{1}{\alpha}-(1+\rho)\right]\left(S_{X}(d)-S_{X}(d+\beta)\right),} & \mathrm{iii},\end{cases} \\
& \frac{\partial \mathrm{CTE}_{T}}{\partial \beta}= \begin{cases}-1+(1+\rho) S_{X}(d+\beta), & \mathrm{i}, \\
(1+\rho) S_{X}(d+\beta)+\frac{f_{X}(d+\beta)}{S_{X}(d+\beta)} \mathrm{CVaR}_{T}^{\mathrm{ii}}-1, & \mathrm{ii},\end{cases}
\end{aligned}
$$


In district ii, the partial derivatives in $d$ and $\beta$ depend on risk distribution $f_{X}$, which makes it difficult to determine the existence or location of the optimal solution. We therefore let

$$
\begin{aligned}
H(\beta)= & \left.\mathrm{CTE}_{T}^{\mathrm{ii}}\right|_{d+\beta=S_{X}^{-1}(\alpha)}-\left.\mathrm{CTE}_{T}^{\mathrm{iii}}\right|_{d \rightarrow S_{X}^{-1}(\alpha)} \\
= & {\left[\frac{1}{\alpha}-(1+\rho)\right] \int_{S_{X}^{-1}(\alpha)}^{S_{X}^{-1}(\alpha)+\beta} S_{X}(x) d x } \\
& +(1+\rho) \int_{S_{X}^{-1}(\alpha)-\beta}^{S_{X}^{-1}(\alpha)} S_{X}(x) d x-\beta .
\end{aligned}
$$

We can obtain the solution $d^{\mathrm{CTE}}$ of $\partial \mathrm{CTE}_{T}^{\mathrm{ii}} / \partial d=0$ with a numerical method (but it may not be in district ii). With (35), determining the position and uniqueness of $d^{\mathrm{CTE}}$ is difficult.

Lemma 6. For a given $\beta(\Theta=\{d \mid d>0\})$ under the condition of (20), CTE can obtain the optimal value in district ii if one of the following conditions is reached:

(1) $H(\beta) \leq 0$;

(2) $d^{C T E} \in$ ii and $\operatorname{CTE}\left(d^{C T E}, \alpha, \beta\right) \leq \operatorname{CTE}_{T}^{i i i}\left(S_{X}^{-1}(\alpha), \alpha, \beta\right)$.

Proof. $\partial \mathrm{CTE}_{T}^{\mathrm{i}} / \partial d<0$, so no optimal solution exists in district i. From (35), we know no optimal solution exists under the condition of (20) because $\mathrm{CTE}_{T}^{\mathrm{iii}}$ is monotonically increasing in $d$. Thus, the optimal solution can only appear in district ii. For $H(\beta) \leq 0$, the left border of district ii is not greater than the left boundary of district iii; hence, no optimal value exists in district ii. As (35) shows, $\partial \mathrm{CTE}_{T}^{\mathrm{ii}} / \partial d$ is continuous. Under the condition of statement (2) in Lemma 6, we can ensure the existence of the optimal solution.

Lemma 7. Under the condition of (20), CTE $E_{T}$ cannot attain the optimal value on givend $(\Theta=\{\beta \mid \beta>0\})$.

Proof. According to (20), $\partial \mathrm{CTE}_{T}^{\mathrm{iii}} / \partial \beta<0$; no optimal value exists in the open set district iii. According to (36), we have

$$
\frac{\partial^{2} \mathrm{CTE}_{T}^{\mathrm{i}}}{\partial \beta^{2}}=-(1+\rho) f_{X}(d+\beta)<0,
$$

where $\mathrm{CTE}_{T}^{\mathrm{i}}$ is strictly convex and the minimum value can only be obtained at the border. However, district $i$ is an open set, so no optimal value exists. When $\beta=\infty$ and $0<d<$ $S_{X}^{-1}(\alpha)$, this is as described by Cai et al. [7]. Therefore,

$$
\mathrm{CTE}_{T}^{\mathrm{ii}}(d, \alpha, \infty)=d+(1+\rho) \int_{d}^{\infty} S_{X}(x) d x .
$$

Moreover, according to (33), we have

$$
\begin{aligned}
\operatorname{CTE}_{T}^{\mathrm{ii}}(d, \alpha, \beta) \\
=\mathrm{CTE}_{T}^{\mathrm{ii}}(d, \alpha, \infty) \\
\quad+\left[\frac{1}{S_{X}(d+\beta)}-(1+\rho)\right] \int_{d+\beta}^{\infty} S_{X}(x) d x .
\end{aligned}
$$

Under (20), $\operatorname{CTE}_{T}^{\mathrm{ii}}(d, \alpha, \beta)>\mathrm{CTE}_{T}^{\mathrm{ii}}(d, \alpha, \infty)$, and no optimal solution exists. $d$ is increasing within district iii, and the minimum can only appear when $d \rightarrow S_{X}^{-1}(\alpha)$. Thus, no optimal solution exists in the open set district iii. When $\beta=\infty$, Cai et al. [7] note that $\mathrm{CTE}_{T}$ is continuous. Under the conditions of (20), the minimum is obtained at $\tilde{d}$ :

$$
\operatorname{CTE}_{T}^{\mathrm{ii}}(\widetilde{d}, \alpha, \infty)=\widetilde{d}+(1+\rho) \int_{\tilde{d}}^{\infty} S_{X}(x) d x .
$$

$\operatorname{CTE}_{T}^{\mathrm{i}}(0, \alpha, \beta)$ is convex on $\beta \in\left(0, S_{X}^{-1}(\alpha)\right)$, so the minimum appears at both ends:

$$
\begin{aligned}
\operatorname{CTE}_{T}^{\mathrm{i}}\left(0, \alpha, S_{X}^{-1}(\alpha)\right) \\
=(1+\rho) \int_{0}^{S_{X}^{-1}(\alpha)} S_{X}(x) d x+\frac{1}{\alpha} \int_{S_{X}^{-1}(\alpha)}^{\infty} S_{X}(x) d x \\
=\mathrm{CTE}_{T}^{\mathrm{ii}}(0, \alpha, \infty) \\
\quad+\left[\frac{1}{\alpha}-(1+\rho)\right] \int_{S_{X}^{-1}(\alpha)}^{\infty} S_{X}(x) d x .
\end{aligned}
$$

Therefore, under the condition of $(20), \operatorname{CTE}_{T}^{\mathrm{i}}\left(0, \alpha, S_{X}^{-1}(\alpha)\right)<$ $\mathrm{CTE}_{T}^{\mathrm{ii}}(0, \alpha, \infty)$. We let

$$
\begin{aligned}
G(d) & =\mathrm{CTE}_{T}^{\mathrm{ii}}(d, \alpha, \infty)-\mathrm{CTE}_{T}^{\mathrm{i}}(d, \alpha, 0), \\
G^{\prime}(d) & =1-(1+\rho) S_{X}(d), \\
G^{\prime \prime}(d) & =(1+\rho) f_{x}(d)>0,
\end{aligned}
$$

where $G$ is convex and $G^{\prime}(\tilde{d})=0 . G\left(S_{X}^{-1}(\alpha)\right)=0$, so $G(\tilde{d})<0 . \mathrm{CTE}_{T}(d, \alpha, 0)$ is a constant, so $\mathrm{CTE}_{T}^{\mathrm{ii}}(\tilde{d}, \alpha, \infty)<$ $\operatorname{CTE}_{T}^{\mathrm{i}}(d, \alpha, 0) . \mathrm{CTE}_{T}^{\mathrm{ii}}(\tilde{d}, \alpha, \infty)$ is the minimum boundary value; if a smaller internal value exists in the flat areas, we can obtain the global optimal solution.

Lemma 8. No global optimum CTE $E_{T}$ exists under the condition of (20), $\Theta=\{(d, \beta) \mid d>0, \beta>0\}$.

Proof. Assume that the optimal solution of $\mathrm{CTE}_{T}$ exists. According to our previous analysis, the global optimal solution can only appear in district ii, and it cannot be the right boundary. We must therefore prove that it is impossible to obtain the optimal value in the lower boundary of district ii:

$$
\begin{aligned}
& \operatorname{CTE}_{T}^{\mathrm{ii}}\left(d, \alpha, S_{X}^{-1}(\alpha)-d\right) \\
& =d+(1+\rho) \int_{d}^{S_{X}^{-1}(\alpha)} S_{X}(x) d x \\
& \quad+\frac{1}{\alpha} \int_{S_{X}^{-1}(\alpha)}^{\infty} S_{X}(x) d x \\
& \operatorname{CTE}_{T}^{\mathrm{ii}^{\prime}}\left(d, \alpha, S_{X}^{-1}(\alpha)-d\right)=1-(1+\rho) S_{X}(d), \\
& \operatorname{CTE}_{T}^{\mathrm{ii}^{\prime \prime}}\left(d, \alpha, S_{X}^{-1}(\alpha)-d\right)=(1+\rho) f_{X}(d)>0 .
\end{aligned}
$$

In the lower boundary, we obtain the optimum at $\tilde{d}$. This is equivalent to having $d$ given; according to Lemma 7 , the above conclusions are reached. 
Assuming that the optimal solution is in the interior of district ii, it must be a saddle point according to the extreme value theory of multivariate functions. Moreover,

$$
\begin{aligned}
& \frac{\partial \mathrm{CTE}_{T}^{\mathrm{ii}}}{\partial \beta}=0, \\
& \frac{\partial \mathrm{CTE}_{T}^{\mathrm{ii}}}{\partial d}=\frac{\partial \mathrm{CTE}_{T}^{\mathrm{ii}}}{\partial \beta}+1-(1+\rho) S_{X}(d)=0 .
\end{aligned}
$$

The solution to the equations is $d=\tilde{d}$, assuming that $\beta=\beta^{\prime}$. This is in the interior of district ii, so $\beta^{\prime}>S_{X}^{-1}(\alpha)-\tilde{d}$. CTE $_{T}$ can only obtain the optimal value in $\left(\tilde{d}, \beta^{\prime}\right)$. However,

$$
\begin{aligned}
\operatorname{CTE}_{T}^{\mathrm{ii}}\left(\tilde{d}, \alpha, \beta^{\prime}\right) \\
=\operatorname{CTE}_{T}^{\mathrm{ii}}(\tilde{d}, \alpha, \infty) \\
\quad+\left[\frac{1}{S_{X}\left(\tilde{d}+\beta^{\prime}\right)}-(1+\rho)\right] \int_{\tilde{d}+\beta^{\prime}}^{\infty} S_{X}(x) d x .
\end{aligned}
$$

In addition, $\tilde{d}+\beta^{\prime}>S_{X}^{-1}(\alpha)$, so $1 / S_{X}\left(\tilde{d}+\beta^{\prime}\right)>(1+\rho)$; that is, $\operatorname{CTE}_{T}^{\mathrm{ii}}\left(\tilde{d}, \alpha, \beta^{\prime}\right)>\mathrm{CTE}_{T}^{\mathrm{ii}}(\tilde{d}, \alpha, \infty)$. No global optimum exists.

\section{Numerical Examples}

5.1. VaR, TVaR, and CTE. We assume that the claim ratio of some insurance is gamma $(4.1405,0.1796)$ and $\alpha=0.1$ and $\rho=0.3$. Figures 5,6 , and 7 show the calculation results of $\mathrm{VaR}, \mathrm{TVaR}$, and CTE, respectively.

Note in Figure 5 that the contour shows a circumstance where the optimal trajectories of a given $d$ and a given $\beta$ cross inside the plane; the crossing point is the global optimal point. In this case, $\widetilde{d^{*}}<0$; this appears when $d$ is fixed on a line of $d+\beta=S_{X}^{-1}(\alpha)$.

Then, as shown in the contour map in Figure 6 and consistent with the theoretical analysis for a given $d$, TVaR decreases with an increase in $\beta$; in other words, no global optimal value exists. For a given $\beta$, a local optimum exists in district ii.

Figure 7 shows that a jump occurs when $d=S_{X}^{-1}(\alpha)$, forming a cross section. However, based on Lemma 6 , the graphs do not show the optimal trajectories to a given $\beta$.

\subsection{Difference between CTE and TVaR. Equations (8)-(13)} show several differences in the denominators in district ii in the function of CTE and TVaR. Even small differences can lead to issues. However, no differences exist between CTE and TVaR when the variable of risk $X$ is continuous; hence, many other studies use them interchangeably. Cai et al. [7] prove that $\mathrm{CTE}_{T}$ is continuous of total loss after reinsurance under stop-loss reinsurance. Thus, they can also be used similarly as substitutes for each other. We prove that $\mathrm{CTE}_{T}$ is discontinuous of total loss after reinsurance under limited stop-loss reinsurance. Obviously, therefore, they cannot be used interchangeably.
We assume that the claim ratio of some insurance is gamma $(4,0.125)$. We have $\rho=0.2, \alpha=0.01$, given reinsurance coverage $\beta=1$. The results imply that TVaR is optimal when $d=0.4317\left(d<S_{X}^{-1}(\alpha)-\beta\right.$, in i), and CTE is optimal when $d=S_{X}^{-1}(\alpha)$, as shown in Figure 8. $\beta=1$ and the franchise point are large under CTE and can almost be approximated by stop-loss reinsurance.

For the risk distribution discussed above, note that (20) remains valid when $\rho=0.5, \alpha=0.2$, and $\beta=0.6$. However, $H(\beta)>0, \operatorname{CTE}(d, \alpha, \beta)>\operatorname{CTE}_{T}^{\mathrm{iii}}\left(S_{X}^{-1}(\alpha), \alpha, \beta\right)$, and $\forall d \in$ ii. Hence, we cannot obtain the optimal solution under CTE. From Lemma 4, we can obtain it under TVaR, $d=0.4152$ $(\in \mathrm{ii})$, which is shown in Figure 8.

The model definition indicates that the CTE risk measures are more accurate than those of TVaR. However, the analysis and examples above show that discontinuous CTE can hinder optimal decision making to some extent.

5.3. Reinsurance Premium Budget Constraints. We mentioned the optimization problem regarding reinsurance premium budget constraints briefly during the introduction. Porth et al. [14] avoid solving the reinsurance premium equation (4), but they used the stochastic simulation method to solve the optimization problem. The previous proof shows that, in some cases, no possible optimal solution exists. Therefore, we first need to determine whether that is the case before applying stochastic simulation.

The CTE in Porth et al. [14] is consistent with TVaR used in this paper. Under financial constraints, whether a local optimum exists depends on the existence of solutions to the equations of $d$ and $\beta$ :

$$
\begin{array}{r}
1-\left[\frac{1}{\alpha}-(1+\rho)\right] S_{X}(d+\beta)-(1+\rho) S_{X}(d)=0, \\
(1+\rho) \int_{d}^{d+\beta} S_{X}(x) d x=\pi .
\end{array}
$$

The first equation derives from (31); as per Lemma 4, we know that this is the local optimal trajectory. The second equation is the financial constraint equation (strict expression is $\delta(d, \beta) \leq \pi)$,

$$
\begin{aligned}
\frac{d d}{d \beta} & =-\frac{[1 / \alpha-(1+\rho)] f_{X}(d+\beta)}{(1+\rho) f_{X}(d)+[1 / \alpha-(1+\rho)] f_{X}(d+\beta)} \\
& <0, \\
\frac{d d}{d \beta} & =\frac{S_{X}(d+\beta)}{S_{X}(d)-S_{X}(d+\beta)}>0 .
\end{aligned}
$$

For the first equation, we have

$$
\beta \longrightarrow \infty \Longrightarrow S_{X}(d+\beta)=0 \Longrightarrow d_{1}=S_{X}^{-1}\left(\frac{1}{1+\rho}\right) \text {. }
$$




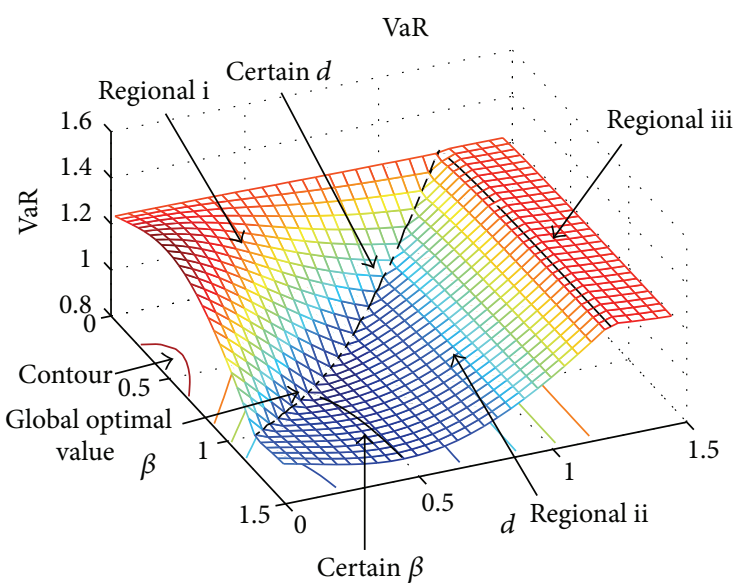

Schematic of the $\mathrm{VaR}_{T}$ value

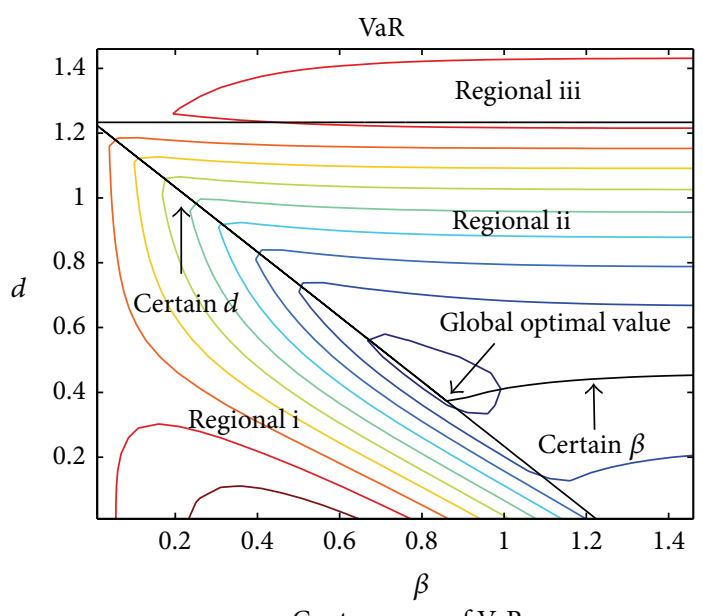

Contour map of $\mathrm{VaR}_{T}$

Figure 5: $\operatorname{VaR}_{T}$ that satisfies (20).

$\mathrm{TVaR}_{T}$

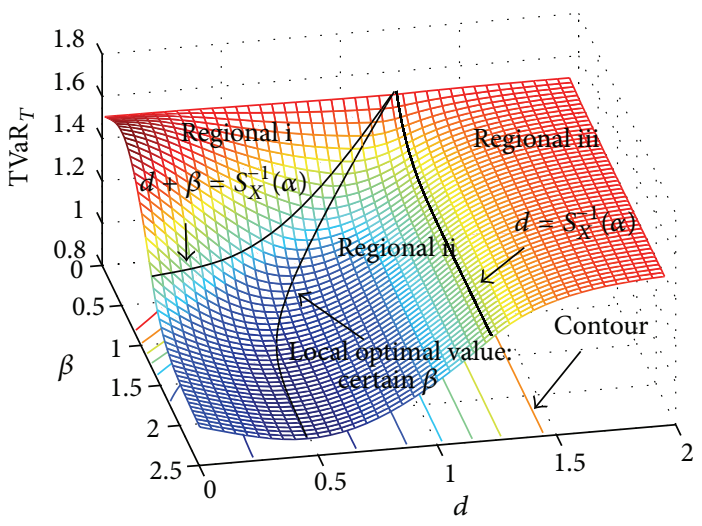

Schematic of $\mathrm{TVaR}_{T}$

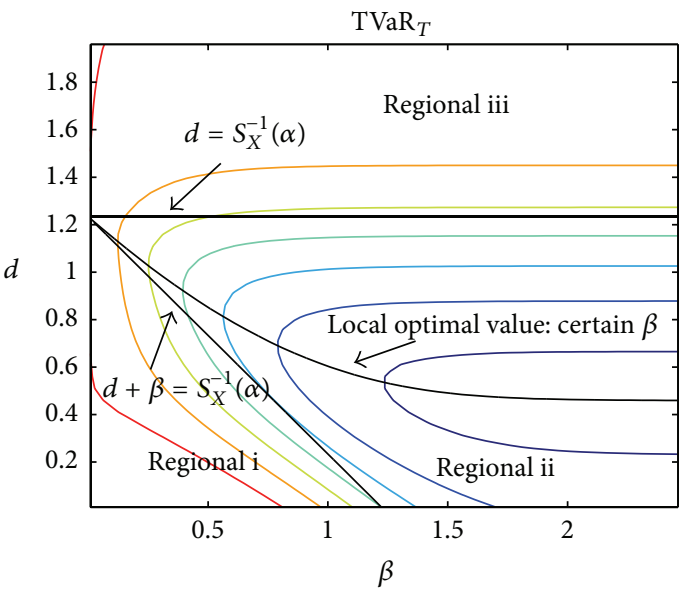

Contour map of $\mathrm{TVaR}_{T}$

Figure 6: $\mathrm{TVaR}_{T}$ that satisfies (20).

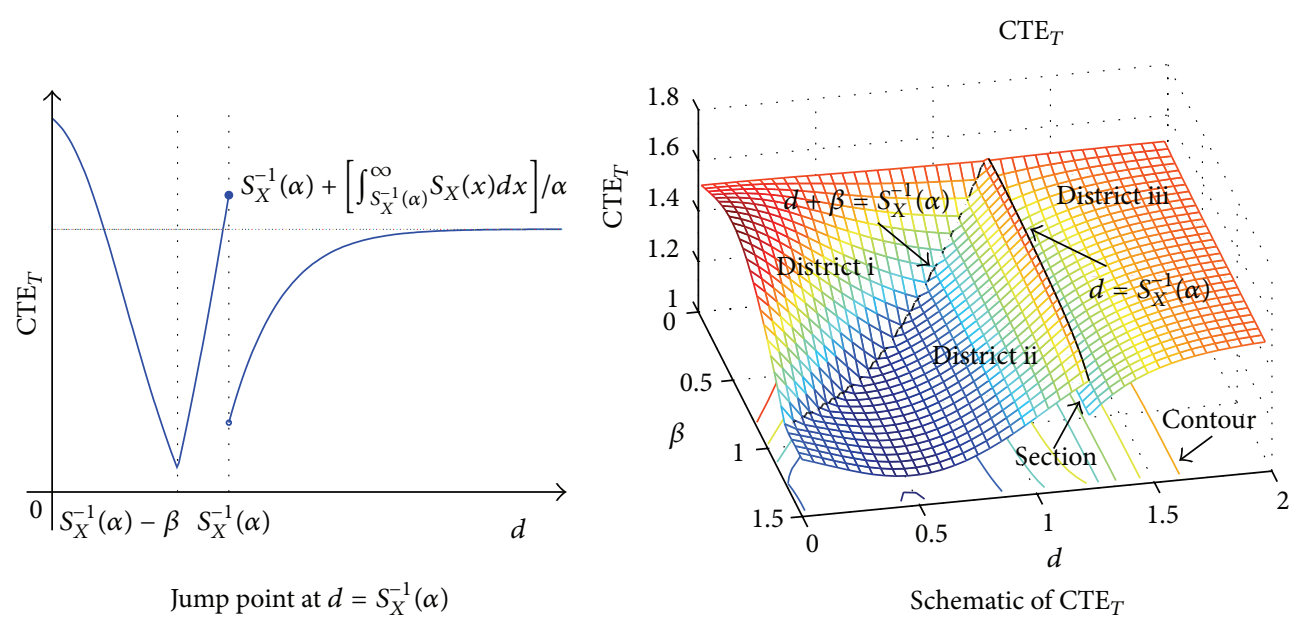

Figure 7: $\mathrm{CTE}_{T}$ that satisfies (20). 

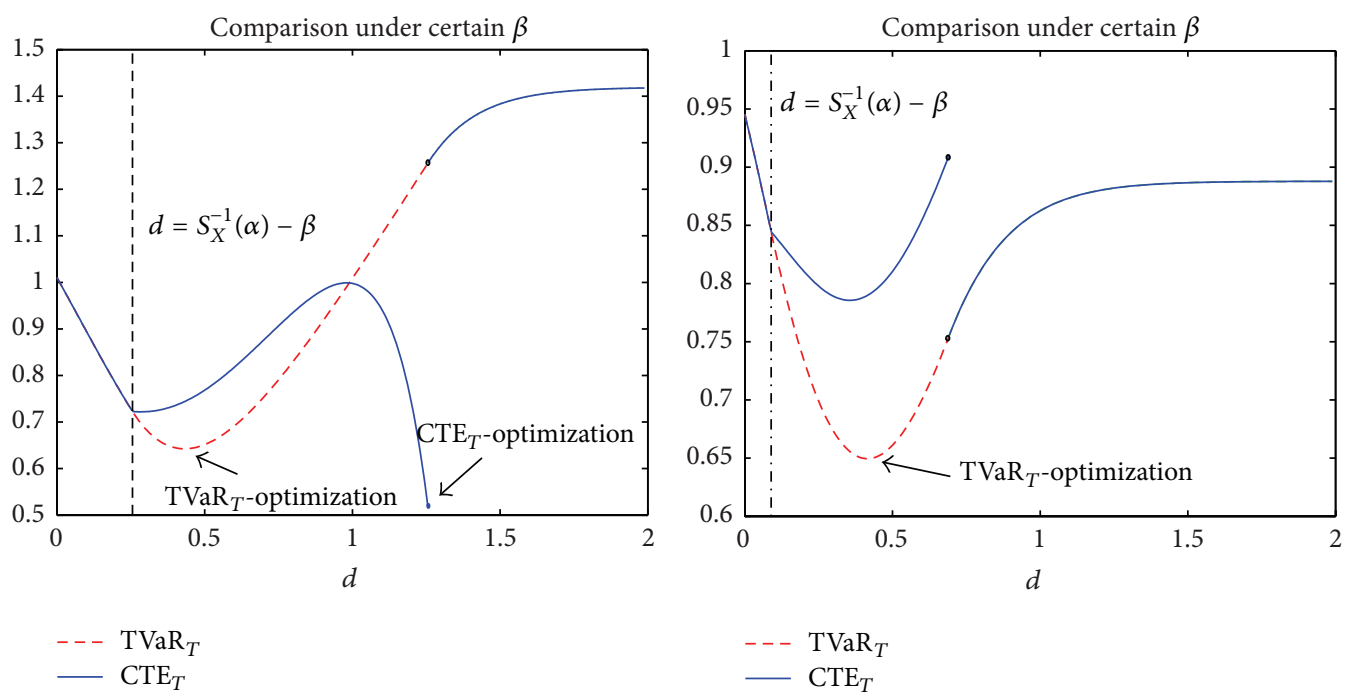

$X \sim \operatorname{gamma}(4,0.125)$ and $\rho=0.2$,
$\alpha=0.01, \beta=1$

$X \sim \operatorname{gamma}(4,0.125)$ and $\rho=0.5$,
$\alpha=0.2, \beta=0.6$

FIGURE 8: Optimal $d$ for given $\beta$.

Under financial constraints, the existence of a local optimal solution depends on whether the following inequality is true:

$$
\begin{aligned}
& \delta\left(S_{X}^{-1}\left(\frac{1}{1+\rho}\right), \infty\right)=(1+\rho) \int_{S_{X}^{-1}(1 /(1+\rho))}^{\infty} S_{X}(x) d d \\
& \quad \leq \pi
\end{aligned}
$$

Similarly, we assume that the claim ratio of some insurance is gamma $(4.1405,0.1796)$ and $\alpha=0.1$ and $\rho=0.3$. However, financial budget $\pi=0.1$ and 0.3 , respectively. The results are shown in Figure 9. " $\pi=0.1$ " is the corresponding financial constraint line. When $\pi=0.1$, the financial constraint line and the local optimal trajectories will effectively intersect (namely, the efficient solution of (47)). When $\pi=0.3$, no intersection point will exist with local optimal trajectories. Therefore, using a numerical solution or stochastic simulation when $\pi=0.3$ will lead to unpredictable results. Although $\pi=0.3$ is therefore not feasible in general in businesses, we cannot rule out its existence in special businesses.

\section{Conclusion}

We have mentioned the numerical solution of the equation several times throughout this paper. But it is ultimately unnecessary. For example, for a given $\beta, \beta^{*}$ is the numerical solution of $h(\beta)=0$ under VaR. To calculate it, we need only to determine whether (18) is positive or negative with $\beta$ rather than with $\beta^{*}$. If we apply the optimization method directly to obtain an optimal solution, then the algorithm discussed here may create some confusion as follows. (1) Is the solution optimal? (2) If not, is that because of the algorithm or is it because no optimal solution exists? On the basis of the numerical method, we posit that the existence of the

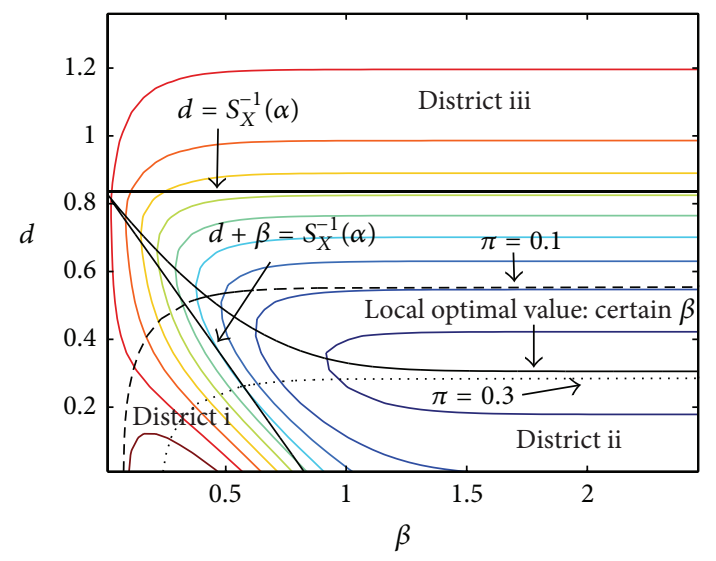

FiguRE 9: Under different financial constraints of $\mathrm{TVaR}_{T}$.

solution and considerable useful location information are theoretically provided.

Compared with stop-loss reinsurance $(\beta=\infty)$, limited stop-loss coverage is more suitable for, for example, agricultural insurance. The latter's limited coverage $\beta$ results in the discontinuity of risk probability functions and risk measures, such as the jump point between $S_{I}$ and the CTE section. This discontinuous district determines the existence and location of an optimal solution.

Table 1 illustrates these results. All discussions are based on (20). Other conditions are also listed in Table 1: "No" means the solution only needs to satisfy (20); "--" means no optimal solution exists (no rigorous solution occurs; e.g., $\beta=\infty)$. Note that VaR does not meet the consistency requirement. However, when we make an optimal reinsurance decision under VaR, it provides clear advice (i.e., global optimal reinsurance arrangements). VaR is limited in that 
TABLE 1: Existence condition of optimal solution.

\begin{tabular}{lccc}
\hline $\begin{array}{l}\text { Risk } \\
\text { measure }\end{array}$ & Certain $\beta$ & Certain $d$ & $\begin{array}{c}\text { Comprehensive } \\
\text { effect }\end{array}$ \\
\hline VaR & $\forall \beta \in\left(0, \beta^{*}\right)$ & $\forall d \in\left[\widetilde{d}^{*}, S_{X}^{-1}(\alpha)\right]$ & No \\
TVaR & $\forall \beta \in\left(0, \beta^{*}\right)$ & - & - \\
CTE & Lemma 6 & - & - \\
\hline
\end{tabular}

the appetite for risk $\alpha$ cannot affect the optimal franchise point, which is determined solely by risk distribution and safety loading. Compared with VaR and TVaR, CTE is more reasonable. However, optimal reinsurance decisions can be difficult to be designed under CTE, and they can only be provided within a limited range. When $\beta$ is given, optimal reinsurance purchase advice can be provided under TVaR and CTE. If risk preference $\alpha$ is known or if the maximum acceptable loss is $d$, then VaR should be the first choice. If the goal of reinsurance coverage is known, CTE would be recommended. In contrast, $\mathrm{ES}$ and $\mathrm{CVaR}$ are inappropriate choices for making reinsurance decisions.

In this study, note that safety loading $\rho$ is a constant. An increase in the reinsurance compensation point will concurrently increase model uncertainty. Thus, we should set $\rho$ as an increasing function of compensation point $d$. Moreover, we considered no financial constraints in this study. However, if we can effectively solve the reinsurance premium equation, then we can also effectively solve the problem of financial constraints and obtain the corresponding analytical solution. Restricted to research methods, we used several numerical methods to determine the existence of an optimal value. Fixed point theory has a strong advantage in dealing with optimization problems. Thus, introducing a fixed point method would avoid the numerical solutions used here.

\section{Conflict of Interests}

The authors declare that there is no conflict of interests regarding the publication of this paper.

\section{Acknowledgments}

This paper was funded by the Youth Project of the National Natural Science Foundation of China (71102125) and the MOE Project of the Key Research Institute of Humanities and Social Sciences at Universities (13JJD790041). The authors are grateful for the support provided by the Beijing Education Committee through the Young Talents Plan Project.

\section{References}

[1] K. Borch, "The safety loading of reinsurance premiums," Scandinavian Actuarial Journal, no. 3-4, pp. 163-184, 1960.

[2] H. U. Gerber, An Introduction to Mathematical Risk Theory, vol. 8, SS Huebner Foundation for Insurance Education, Wharton School, University of Pennsylvania, Philadelphia, Pa, USA, 1979.

[3] L. Gajek and D. Zagrodny, "Insurer's optimal reinsurance strategies," Insurance: Mathematics \& Economics, vol. 27, no. 1, pp. 105-112, 2000.
[4] M. Kaluszka, "Mean-variance optimal reinsurance arrangements," Scandinavian Actuarial Journal, no. 1, pp. 28-41, 2004.

[5] Y. Bu, "On optimal reinsurance arrangement," in Casualty Actuarial Society Forum, pp. 1-20, 2005.

[6] J. Cai and K. S. Tan, "Optimal retention for a stop-loss reinsurance under the VaR and CTE risk measures," Astin Bulletin, vol. 37, no. 1, pp. 93-112, 2007.

[7] J. Cai, K. S. Tan, C. Weng, and Y. Zhang, "Optimal reinsurance under VaR and CTE risk measures," Insurance: Mathematics \& Economics, vol. 43, no. 1, pp. 185-196, 2008.

[8] C. Weng, Optimal reinsurance designs: from an insurer's perspective [Ph.D. thesis], University of Waterloo, 2009.

[9] L. Fu and C. Khury, "Optimal layers for catastrophe reinsurance," Variance, vol. 4, no. 2, pp. 191-208, 2010.

[10] Y. Chi and K. S. Tan, "Optimal reinsurance under VaR and CVaR Risk measures: a simplified approach," Astin Bulletin, vol. 41, no. 2, pp. 487-509, 2011.

[11] Y. Chi and C. Weng, "Optimal reinsurance subject to vajda condition," Insurance: Mathematics and Economics, vol. 53, no. 1, pp. 179-189, 2013.

[12] L. Gajek and D. Zagrodny, "Optimal reinsurance under general risk measures," Insurance: Mathematics and Economics, vol. 34, no. 2, pp. 227-240, 2004.

[13] M. Guerra and M. L. Centeno, "Optimal reinsurance policy: the adjustment coefficient and the expected utility criteria," Insurance: Mathematics \& Economics, vol. 42, no. 2, pp. 529-539, 2008.

[14] L. Porth, K. S. Tan, and C. Weng, "Optimal reinsurance analysis from a crop insurer's perspective," Agricultural Finance Review, vol. 73, no. 2, pp. 310-328, 2013.

[15] Q. Li, M. Gu, and Z. Liang, "Optimal excess-of-loss reinsurance and investment polices under the CEV model," Annals of Operations Research, vol. 223, no. 1, pp. 273-290, 2014.

[16] M. Brandtner and W. Kürsten, "Solvency ii, regulatory capital, and optimal reinsurance: how good are conditional value-atrisk and spectral risk measures?" Insurance: Mathematics and Economics, vol. 59, pp. 156-167, 2014.

[17] C. Bernard and W. Tian, "Optimal reinsurance arrangements under tail risk measures," Journal of Risk and Insurance, vol. 76, no. 3, pp. 709-725, 2009.

[18] P. Artzner, F. Delbaen, J.-M. Eber, and D. Heath, "Coherent measures of risk," Mathematical Finance, vol. 9, no. 3, pp. $203-$ 228, 1999.

[19] T. Hang, Actuarial Aspects of Non-Life Insurance, China Financial \& Economic Publishing House, Beijing, China, 2010.

[20] A. Charpentier, Mesures de risque, Université Rennes 1, Rennes, France, 2010.

[21] J. L. Wirch and M. R. Hardy, "A synthesis of risk measures for capital adequacy," Insurance: Mathematics and Economics, vol. 25, no. 3, pp. 337-347, 1999. 


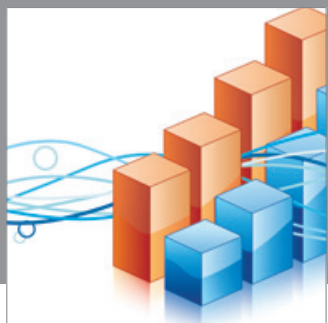

Advances in

Operations Research

mansans

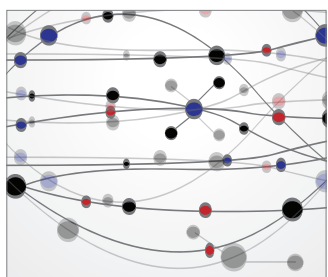

The Scientific World Journal
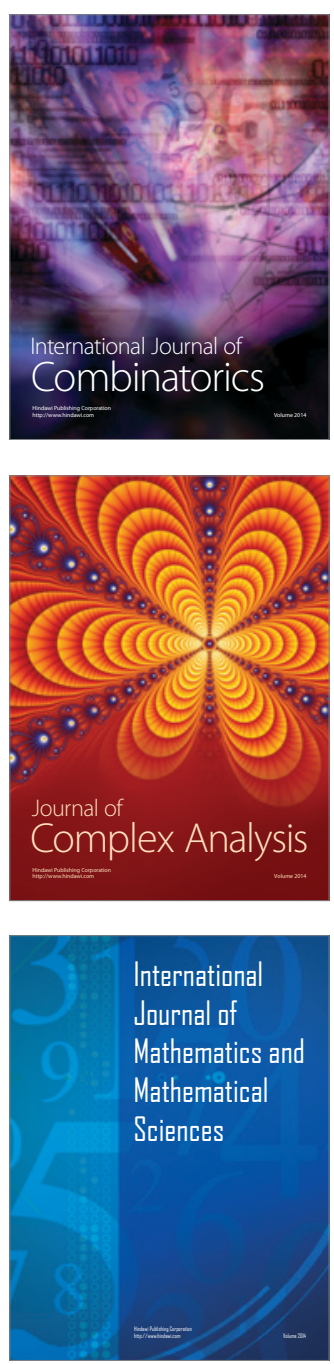
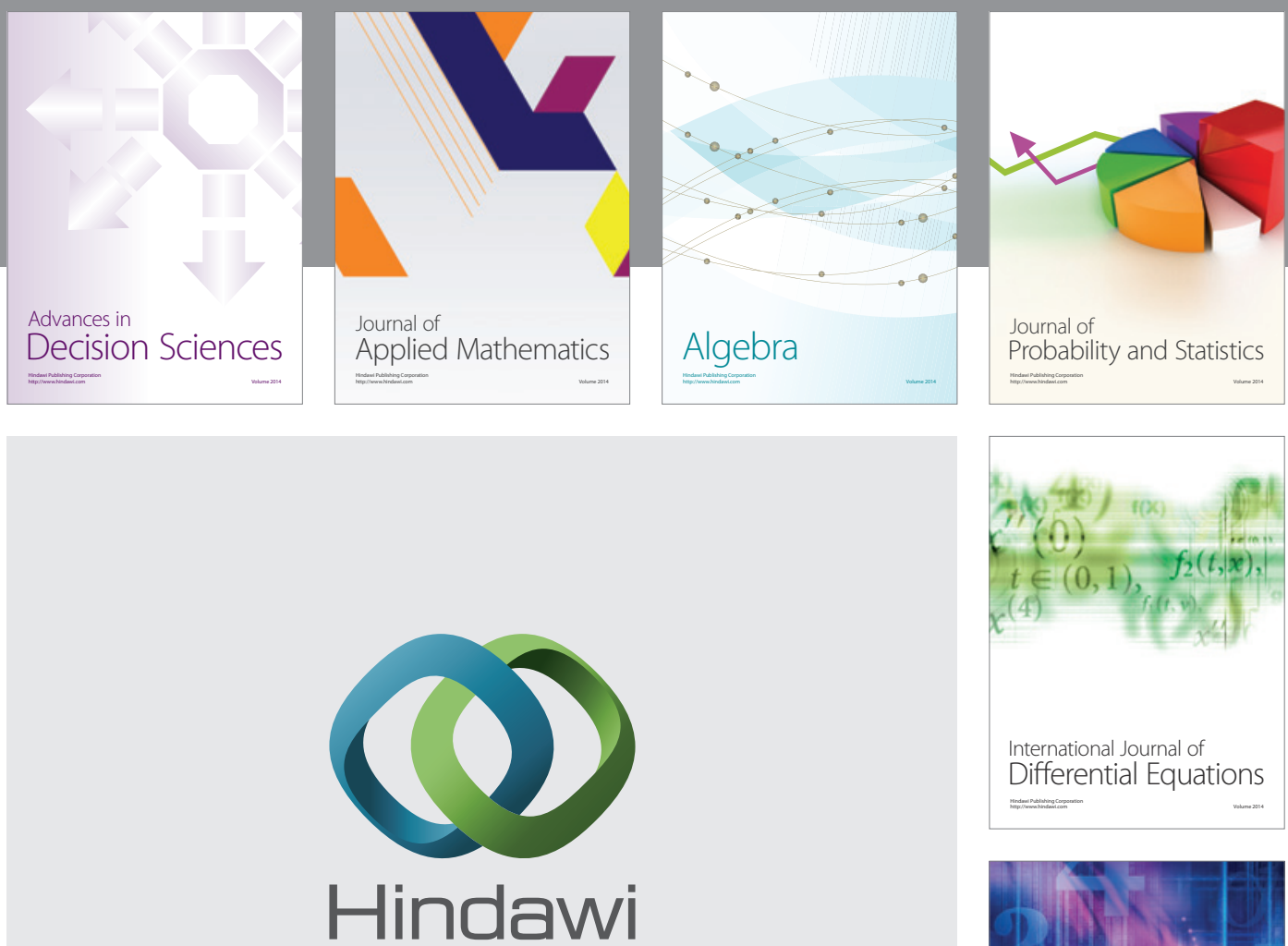

Submit your manuscripts at http://www.hindawi.com
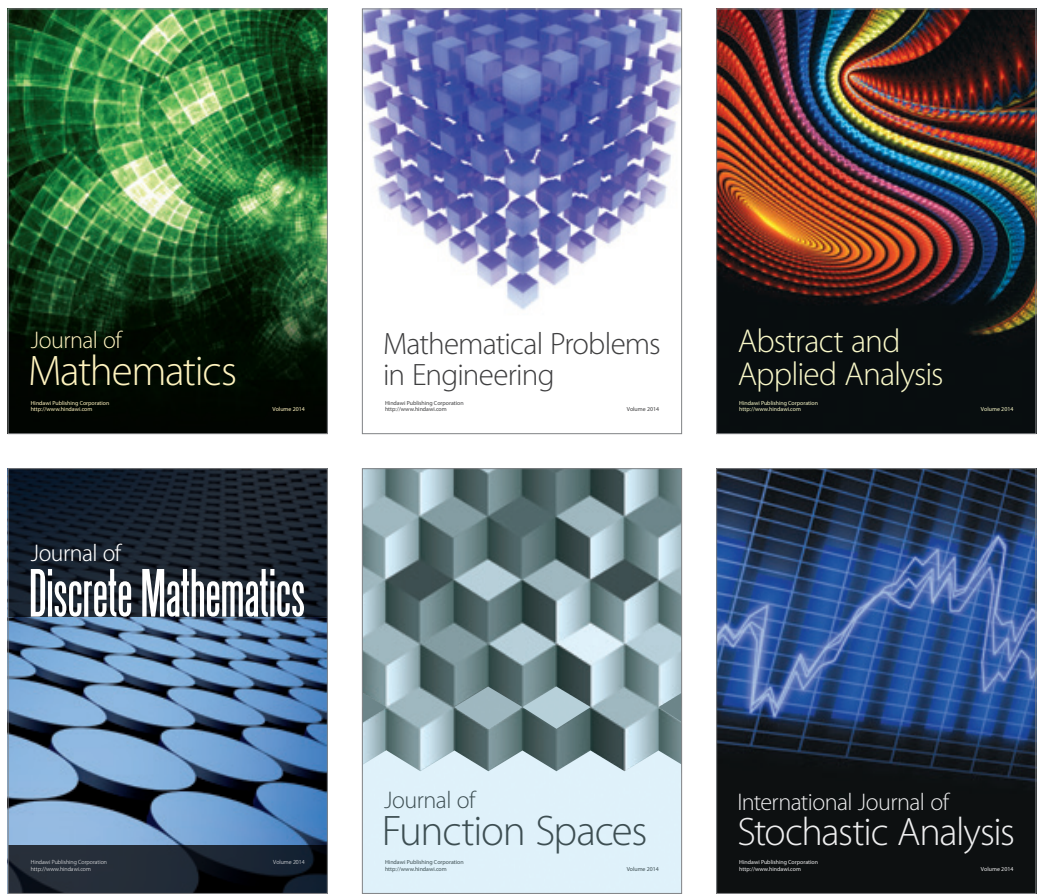

Journal of

Function Spaces

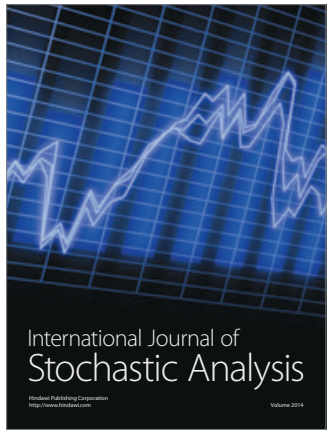

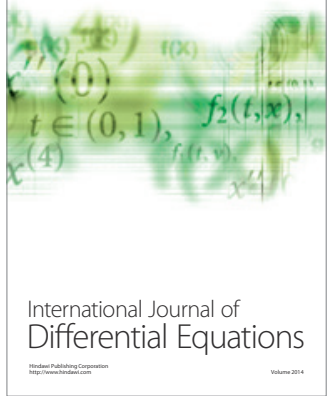
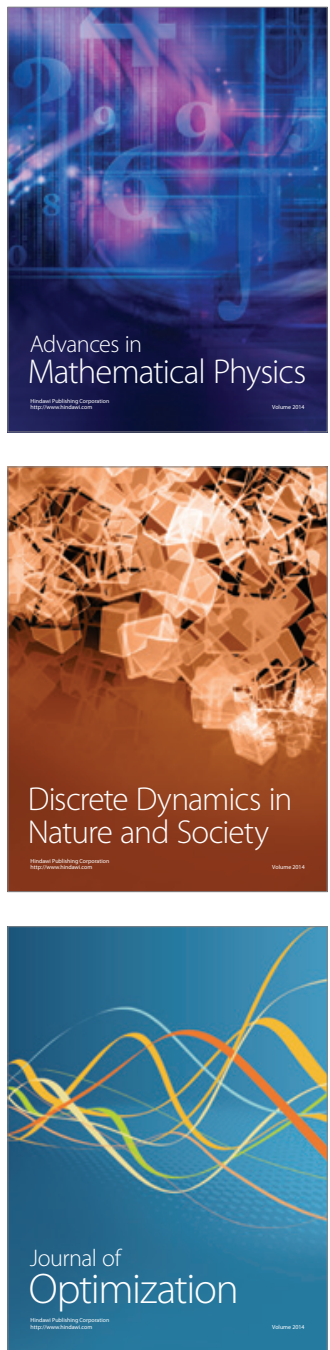